\title{
Neuropathological comparisons of amnestic and nonamnestic mild cognitive impairment
}

\author{
Brittany N. Dugger ${ }^{*}$, Kathryn Davis ${ }^{2}$, Michael Malek-Ahmadi ${ }^{2}$, Joseph G. Hentz ${ }^{3}$, Shawn Sandhu², \\ Thomas G. Beach ${ }^{1}$, Charles H. Adler ${ }^{3}$, Richard J. Caselli ${ }^{3}$, Travis A. Johnson ${ }^{3}$, Geidy E. Serrano ${ }^{1}$, Holly A. Shill², \\ Christine Belden ${ }^{2}$, Erika Driver-Dunckley ${ }^{3}$, John N. Caviness ${ }^{3}$, Lucia I. Sue ${ }^{1}$, Sandra Jacobson ${ }^{2}$, Jessica Powell ${ }^{2}$ \\ and Marwan N. Sabbagh ${ }^{2}$
}

\begin{abstract}
Background: Although there are studies investigating the pathologic origins of mild cognitive impairment (MCl), they have revolved around comparisons to normal elderly individuals or those with Alzheimer's disease (AD) or other dementias. There are few studies directly comparing the comprehensive neuropathology of amnestic (aMCl) and nonamnestic (naMCl) MCl.

Methods: The database of the Brain and Body Donation Program (www.brainandbodydonationprogram.org), a longitudinal clinicopathological study of normal aging and neurodegenerative disorders, was queried for subjects who were carrying a diagnosis of $\mathrm{aMCl}$ or naMCl at the time of autopsy. Neuropathological lesions, including neuritic plaques, neurofibrillary tangles (NFTs), Lewy bodies (LBs), infarcts, cerebral white matter rarefaction (CWMR), cerebral amyloid angiopathy (CAA), and concurrent major clinicopathological diagnoses, including Parkinson's disease (PD) were analyzed.
\end{abstract}

Results: Thirty four subjects with $\mathrm{aMCl}$ and $15 \mathrm{naMCl}$ met study criteria. Subjects with aMCl were older at death (88 vs. 83 years of age, $p=0.03)$. Individuals with naMCl had higher densities of LBs within the temporal lobe $(p=0.04)$ while subjects with aMCI had a propensity for increased NFTs in parietal and temporal lobes ( $p$ values $=0.07$ ). After adjusting for age at death, the only significant difference was greater densities of temporal lobe NFTs within the $\mathrm{aMCl}$ group. Other regional pathology scores for plaques, NFTs, and LBs were similar between groups. Subjects met clinico-pathological criteria for co-existent PD in $24 \% \mathrm{aMCl}$ and $47 \%$ naMCl while neuropathological criteria for $\mathrm{AD}$ were met in equal percentages of aMCl and of naMCl cases (53\%); these proportional differences were not significant ( $p$ values >0.35). Furthermore, regardless of amnestic status, there was a greater presence of CAA (71\% of $\mathrm{MCl}$ with executive dysfunction vs. $39 \%$ without $p=0.03)$ and a greater presence of CWMR ( $81 \%$ of MCl with executive dysfunction and $54 \%$ without $p=0.046$ ) in $\mathrm{MCl}$ cases with executive dysfunction.

Conclusions: No single pathologic entity strongly dichotomized $\mathrm{MCl}$ groups, perhaps due to the pathologic heterogeneity found within both entities. However, these data suggest the possibility for naMCl to have a propensity for increased LBs and aMCI for increased NFTs in select anatomic regions.

\footnotetext{
* Correspondence: Brittany.dugger@bannerhealth.com

${ }^{1}$ Civin Laboratory for Neuropathology, Banner Sun Health Research Institute, 10515 W. Santa Fe Dr., Sun City, AZ 85351, USA

Full list of author information is available at the end of the article
} 


\section{Background}

Mild cognitive impairment (MCI) is a clinical diagnostic term for elderly subjects with defined forms of cognitive dysfunction that do not meet criteria for dementia [1]. In most subjects, $\mathrm{MCI}$ is an intermediate phase between normal cognition and dementia. The term was first used nearly a quarter of a century ago by Reisberg and colleagues, who observed a progression to dementia in an elderly population with mildly impaired cognitive function [2]. Thirteen years later, the classification of $\mathrm{MCI}$ as proposed by the $2^{\text {nd }}$ International Working Group on MCI Criteria included the subtypes of amnestic MCI (aMCI) and nonamnestic MCI (naMCI) [1, 3]. Memory impairment is the defining feature of aMCI, while naMCI is defined by deficits of other cognitive abilities, including attention, executive function, visuospatial skills, and language. Both aMCI and naMCI may affect single or multiple neuropsychological domains. In 2011, criteria were published focusing on $\mathrm{MCI}$ due to Alzheimer's disease (AD) [4].

Most studies investigating the pathologic origins of $\mathrm{MCI}$ have revolved around comparisons to normal elderly individuals or those with $\mathrm{AD}$ or other dementias [5-13]. There are few studies directly comparing the comprehensive neuropathology of aMCI and naMCI. It is critical to investigate pathological similarities and differences between $\mathrm{aMCI}$ and naMCI given the proposed dichotomous progression of each of these diagnoses- i.e. aMCI progressing more towards $\mathrm{AD}[9,12]$. The purpose of this study is to compare and contrast common neuropathologies in subjects who were diagnosed with aMCI or naMCI.

\section{Methods}

\section{Subjects}

Subjects were selected from the Arizona Study of Aging and Neurodegenerative Disorders (AZSAND) with autopsies in the Brain and Body Donation Program (BBDP) at Banner Sun Health Research Institute in Sun City, Arizona (www.brainandbodydonationprogram.org) [14, 15]. All enrolled subjects or their legal representatives sign a written informed consent approved by an Institutional Review Board (Western Institutional Review Board, Puyallup, WA) before the time of death allowing both clinical assessments during life and several options for brain and/or body organ donation after death. The Institutional Review Board approval covers any studies conducted with data and/or tissues from deceased BBDP subjects. Permission to access the research database with HIPPA compliant de-identified information was granted by the director of the BBDP. Access to the research database is open to investigators who are part of the Arizona Alzheimer's Disease Consortium, the Arizona Parkinson's Disease Consortium, as well as researchers who are registered on the BBDP website (www.brainandbodydonationprogram.org) all of which are approved to access the database under the Institutional Review Board. Annual visits and exams for cognition and movement disorders and criteria for clinical diagnoses are previously described [14]. All assessments were conducted in accordance with the Declaration of Helsinki.

The BBDP database was queried for individuals who were autopsied between January 1997 through July 2014, had at least one formal, standardized BBDP neurological evaluation during life, were carrying a diagnosis of aMCI or naMCI according to published criteria $[1,4]$ at their last visit prior to death, and had a completed neuropathological evaluation. Cases were excluded if they had a diagnosis of dementia or had been thought to have cognitive dysfunction that was secondary to a medical illness. If an individual was not seen within 18 months before death, a standardized telephone interview was conducted with a knowledgeable contact, typically next of kin, to evaluate for the presence of dementia or a movement disorder in the final year of life. Additionally, at least two years of private medical records were available for each subject and these were reviewed to provide supplementary information where necessary.

\section{Clinical assessment}

Prior to death, all participants were assessed annually on a variety of clinical, neuropsychological, and functional measures as described previously [14]. Cognition was assessed using an array of measures, which are detailed in a recent publication of the BBDP [15]. A supervising geriatric psychiatrist and neuropsychologist (SJ and $\mathrm{CB}$, respectively) at the Banner Sun Health Research Institute oversaw administration of these measures. Results of these assessments were jointly interpreted by a geriatric psychiatrist (SJ), behavioral neurologist (MNS, RJC), movement disorders neurologist (CHA, HAS, JNC, ED), and neuropsychologist $(\mathrm{CB})$ in order to reach a consensus diagnosis for each individual. With respect to initial disagreements among the consensus panel, all collateral information available (previous years' assessments, medical records, non-cognitive questionnaires, mood measures, functional assessments, etc.) is reviewed and discussed until a consensus is reached. For the clinical diagnoses of aMCI and naMCI, published criteria were used in which neuropsychological test performance fell at or below 1.5 standard deviations in one or more domains of cognition $[1,4]$.

\section{Neuropathological assessment}

All cases underwent autopsy and had a standardized neuropathological assessment blinded to clinical categorization. The final clinicopathological diagnoses were based on both the neuropathology and clinical characteristics as obtained from standardized neurological assessments as well as review of private medical records. Criteria for these 
diagnoses have been reviewed elsewhere [16, 17]. A clinicopathological diagnosis of Parkinson's disease (PD) was made if the subject had two or more cardinal clinical signs (rest tremor, bradykinesia, rigidity) as well as Lewy bodies and pigmented neuron loss in the substantia nigra. All subjects were genotyped for apolipoprotein E (ApoE) using a modification of a standard method [18].

Tissue processing methods have been previously described [14, 19]. In brief, formaldehyde-fixed $5 \mu \mathrm{m}$ paraffin-embedded sections were stained with hematoxylin and eosin, while large-format, $40-80 \mu \mathrm{m}$ thick formaldehyde-fixed sections were stained for plaques, tangles and other features using Gallyas, Thioflavin$\mathrm{S}$ and Campbell-Switzer methods [20]. All subjects were classified according to the Unified Staging System for Lewy Body (LB) Disorders [21] after immunohistochemical evaluation of phosphorylated $\alpha$-synuclein on $5 \mu \mathrm{m}$ paraffin sections [22]. Individuals with incidental LBs (iLBs) were defined as those lacking signs of parkinsonism and/or dementia during life but with autopsy findings of positive phosphorylated $\alpha$-synuclein-immunoreactive neuronal elements, morphologically consistent with Lewytype pathologies. Semi-quantitative analyses of neuritic plaques (NP) and neurofibrillary tangle (NFT) densities were done using standardized Consortium to Establish a Registry for Alzheimer's Disease (CERAD) published templates [23]; estimates of none, sparse, moderate, or frequent were converted to a continuous 0-3 scale for statistical purposes. Regions scored included cortical gray matter from standard levels of frontal, parietal, and temporal lobes, as well as hippocampal and entorhinal regions. Global NP and NFT scores were obtained by summation of all regions analyzed with a maximum score of 15. For this study, subjects received a pathological diagnosis of $\mathrm{AD}$ if they were classified as "intermediate" or "high" according to the NIA-Reagan criteria, regardless of the absence of dementia [24]; Braak NFT stage was also determined [25]. Cerebral white matter rarefaction (CWMR) was defined as having $26 \%$ or more of the centrum semiovale affected in one or more of the following lobes: frontal, parietal, occipital, and temporal; these methods have been published previously [26]. Cerebral amyloid angiopathy (CAA) was graded by density of amyloidotic blood vessels, using a $0-3$ scale analogous to CERAD templates [23]. Brain neoplasms were defined as primary or metastatic parenchymal tumors and did not include meningiomas or schwannomas. Argyrophilic grains (Arg) were defined as typical spindle-shaped structures revealed by the Gallyas silver stain [27]. Presence/absence of non-acute infarcts were categorized by affected region (cortical, centrum semiovale, deep nuclei, and/or infratentorial regions) and by size (microscopic (not grossly visible), lacunar (<1 cc), small (1-27 cc), and large (>27 cc)). Infarct groups were not mutually exclusive. For evaluation of mixed pathologies- only the three diagnoses of 1) meeting neuropatholgic criteria for AD; 2) cases having LBs in areas other than the olfactory bulb, and 3) presence of non-acute infarcts were considered. These were then divided into cases with none, one, two, or three of the mentioned diagnosis; similar methods have been done previously [28].

\section{Statistical analysis}

Two-tailed, unpaired $t$ tests were used to compare differences between aMCI and naMCI cases on age at death, time from MCI diagnosis to death, years of education, Mini Mental State Examination (MMSE) score and Part III (motor) of the Unified Parkinson's Disease Rating Scale (UPDRS). Pearson chi-square tests were used to determine differences in frequency for neuropathological diagnosis of $\mathrm{AD}$, concurrent major clinicopathological diagnoses, Arg, CAA, CWMR, brain neoplasms, iLBs, infarcts, gender, and ApoE $\varepsilon 4$ carrier status. The Fisher exact test was used instead of the Pearson chi-square test when the minimum expected cell count was less than five. The Wilcoxon rank-sum test was used to compare Braak stage, NP score, and Unified Lewy Body stage. After initial analyses, all data were then adjusted for age at death.

\section{Results}

\section{Overall demographics}

Of the 1185 autopsied BBDP subjects queried, 49 (4\%) met inclusion criteria for aMCI or naMCI. Of these 49 subjects, $20(40 \%)$ were female, mean $( \pm \mathrm{SD})$ age at death was $87 \pm 7.8$ years (range 69-103 years), and the average time from last neurological evaluation to death was $14 \pm 13.3$ months (range 2 to 65 months). Thirty-four (69 \%) met criteria for aMCI, while 15 (31\%) were naMCI.

\section{Differences and similarities between $\mathrm{aMCl}$ and naMCI}

Demographic and pathologic characteristics of the two groups are displayed in Table 1. The aMCI group was significantly older, but there were no large differences with respect to gender ratios, ApoE4 carrier frequency (there were no aMCI or naMCI that were homozygous for ApoE4), time from last neurological (neuro) exam until death, last MMSE score and last UPDRS score. With respect to domains affected, naMCI has significantly greater frequencies of cases with executive dysfunction (67 vs. $32 \%, p=0.03$ ). There were no other large differences with respect to demographic information between aMCI and naMCI (differences less than 22 percentage points or 0.4 standard deviations).

Some subjects had concomitant clinicopathological diagnoses (Table 2), including Parkinson's disease (PD), progressive supranuclear palsy (PSP), motor neuron disease (MND), and multiple system atrophy (MSA); 
Table 1 Demographic information of aMCI and naMCl cases in the BBDP. Mean \pm SD or $n(\%)$

\begin{tabular}{|c|c|c|c|c|}
\hline & $\mathrm{aMCl}(\mathrm{N}=34)$ & $\operatorname{naMCl}(n=15)$ & $95 \% \mathrm{Cl}$ & $P$ value \\
\hline Female (\%) & $12(35 \%)$ & $7(47 \%)$ & -0.41 to 0.19 & 0.53 \\
\hline Multi-domain N (\%) & $13(38 \%)$ & $4(27 \%)$ & -0.16 to 0.39 & 0.43 \\
\hline \multicolumn{5}{|l|}{ Domains affected } \\
\hline Memory & $34(100 \%)$ & $\mathrm{n} / \mathrm{a}$ & $\mathrm{n} / \mathrm{a}$ & $\mathrm{n} / \mathrm{a}$ \\
\hline Executive & $11(32 \%)$ & $10(67 \%)$ & -0.63 to -0.06 & 0.03 \\
\hline Language & $4(12 \%)$ & $5(33 \%)$ & -0.50 to 0.03 & 0.11 \\
\hline Visuospatial & $2(6 \%)$ & $3(20 \%)$ & -0.42 to 0.06 & 0.16 \\
\hline Attention & $1(3 \%)$ & $2(13 \%)$ & -0.36 to 0.07 & 0.22 \\
\hline Age at death, yrs & $88 \pm 8$ & $83 \pm 7$ & 0.4 to 9.8 & 0.03 \\
\hline Interval last neuro exam till death, months & $15 \pm 15$ & $11 \pm 7.7$ & -0.61 to 0.67 & 0.86 \\
\hline Education, yrs. & $15 \pm 3$ & $16 \pm 2$ & -3.3 to 0.0 & 0.05 \\
\hline APOE 4 carriers & $9(26 \%)$ & $6(40 \%)$ & -0.43 to 0.15 & 0.50 \\
\hline Last MMSE & $27 \pm 2$ & $27 \pm 2[n=14]$ & -1.8 to 0.9 & 0.55 \\
\hline Interval last MMSE till death, months & $15 \pm 15$ & $10 \pm 5[n=14]$ & -3.3 to 13.2 & 0.23 \\
\hline Last UPDRS & $20 \pm 16[n=33]$ & $23 \pm 17$ & -13 to 8 & 0.63 \\
\hline Interval last UPDRS till death, months & $15 \pm 15$ & $17 \pm 19$ & -12 to 9 & 0.78 \\
\hline
\end{tabular}

frequencies of these diagnoses were not substantially different between aMCI and naMCI (differences less than 22 percentage points). Subjects met clinicopathological criteria for co-existent PD in $24 \%$ aMCI and $47 \%$ naMCI while neuropathological criteria for $\mathrm{AD}$ were met in equal percentages of aMCI and of naMCI cases (53\%).

All MCI cases had one or more of the following pathologies: neurofibrillary tangles (NFTs), NP, and LBs- the most common being NFTs. All aMCI and naMCI contained at least some NFTs, while 12 (35\%) aMCI and 6 (40\%) naMCI were devoid of NP in all areas examined; categories of age related CERAD NP density scores and Braak NFT staging are listed in Table 2. Both aMCI and naMCI had similar distributions and densities of NP (Table 3). However, for NFTs, there was a propensity for aMCI to have higher densities than naMCI in the temporal and parietal lobe (Bayes posterior probability $96 \%$ ), although this did not reach statistical significance $(P$ values $=0.07$ ). For the presence of Lewy-type alphasynucleinopathy (Table 3), only the temporal lobe was statistically significant $(p=0.04)$ with naMCI having greater densities than aMCI. With respect to overall differences in LBs (i.e. examining just for the presence of LBs irrespective of any parkinsonian features), there were no large differences based on the presence of LBs, with $38 \%$ of aMCI and $43 \%$ of naMCI having LBs (95\% CI: -0.36 to $0.26 p=0.73$ ). After adjusting for age at death, the only large difference was greater temporal lobe NFTs within the aMCI group (95\% CI: 0.00 to $0.69, p=0.05$ ). As for other pathologies, the most common pathologies in aMCI and naMCI were CWMR (65 \% aMCI and $67 \%$ naMCI) and CAA (67 \% aMCI and $47 \%$ naMCI). There were only 2 cases with brain neoplasms both of which had aMCI. There were also similar frequencies with respect to Arg (37\% aMCI, $27 \%$ naMCI) and acute infarcts (18\% aMCI, $13 \%$ naMCI). Frequencies for non-acute infarcts are located in Table 4. The frequency for non-acute infarcts for aMCI was 53 and $40 \%$ for naMCI. There were no significant differences on any infarct measures (location and/or size) between aMCI and naMCI. No case had a pathological diagnosis of hippocampal sclerosis.

With respect to mixed pathologies (infarcts, any LBs, and/or a neuropathologic diagnosis of AD), $20 \%$ of naMCI and $5.9 \%$ of aMCI contained none of the pathologies $(p=0.16$, Fisher's Exact Test), $33.3 \%$ of naMCI and $41.2 \%$ of aMCI contained 1 of the pathologies $\left(x^{2}=\right.$ $0.04 p=0.84), 33.3 \%$ of naMCI and $52.9 \%$ of aMCI contained at 2 of the pathologies $\left(\chi^{2}=0.92 \mathrm{p}=0.34\right)$, and $13 \%$ of naMCI contained all three $(p=0.09$, Fisher's Exact Test) (Fig. 1).

Lastly, with respect to the presence/absence of executive dysfunction (regardless of aMCI or naMCI status) there were some significant differences. There was a significant relationship between the presence of CAA and executive dysfunction, (71 \% of MCI with executive dysfunction and $39 \%$ without executive dysfunction- $95 \%$ CI: 0.06 to 0.59 $p=0.03)$ and also a significant relationship between the presence of CMWR and executive dysfunction (81\% of MCI with executive dysfunction and $54 \%$ without - $95 \%$ CI: 0.02 to $0.52 p=0.046$ ).

\section{Discussion}

The current study demonstrates the vast array of neuropathologic heterogeneity within $\mathrm{MCI}$, suggesting that 
Table 2 Frequencies of pathologies within aMCl $(N=34)$ and naMCl $(N=15)$ cases. All pathology groups are not mutually exclusive; there is considerable overlap with concomitant pathologies

\begin{tabular}{|c|c|c|c|}
\hline & $\mathrm{aMCl}$ & naMCl & $p$ value \\
\hline \multicolumn{4}{|l|}{ Clinicopathologic diagnoses } \\
\hline PD & $8(24 \%)^{a}$ & 7 (47 \%) & 0.18 \\
\hline PSP & $3(9 \%)^{a}$ & $3(20 \%)$ & 0.35 \\
\hline MND & 0 & $1(7 \%)$ & 0.31 \\
\hline MSA & $1(3 \%)$ & 0 & 1.00 \\
\hline \multicolumn{4}{|l|}{ Other pathologies } \\
\hline Met neuropath criteria for AD & $18(53 \%)$ & $8(53 \%)$ & 1.00 \\
\hline Braak NFT stage & & & 0.22 \\
\hline I & $2(6 \%)$ & 0 & \\
\hline$\|$ & $2(6 \%)$ & $2(13 \%)$ & \\
\hline III & $3(9 \%)$ & 5 (33\%) & \\
\hline IV & $23(68 \%)$ & $8(53 \%)$ & \\
\hline V & $3(9 \%)$ & 0 & \\
\hline $\mathrm{Vl}$ & $1(3 \%)$ & 0 & \\
\hline CERAD NP score & & & 0.90 \\
\hline None & $12(35 \%)$ & $6(40 \%)$ & \\
\hline Sparse & $2(6 \%)$ & $1(7 \%)$ & \\
\hline Moderate & $8(24 \%)$ & $2(13 \%)$ & \\
\hline Frequent & $12(35 \%)$ & $6(40 \%)$ & \\
\hline Incidental LBs & $5(15 \%)$ & 0 & 0.31 \\
\hline Unified LB staging scheme & & & 0.18 \\
\hline Stage 0 . no LBs & $21(62 \%)$ & $8(53 \%)$ & \\
\hline Stage 1. OBT only & $2(6 \%)$ & 0 & \\
\hline Stage Ila. Brainstem & $3(9 \%)$ & $1(7 \%)$ & \\
\hline Stage Illb. Limbic & $3(9 \%)$ & 0 & \\
\hline Stage III. Limbic + Brainstem & $3(9 \%)$ & $3(20 \%)$ & \\
\hline Stage IV. Neocortical & $2(6 \%)$ & $3(20 \%)$ & \\
\hline
\end{tabular}

${ }^{\mathrm{a}}$ One aMCl case had both PSP and PD; there were no other overlapping clinicopathological diagnoses

there may be no single underlying etiology dichotomizing aMCI from naMCI. This is supported by previous studies demonstrating multiple pathologies are found to be present in MCI $[5,6,10,29]$. This study differs from most previous studies by directly comparing neuropathology in two main subtypes of MCI, i.e. aMCI and naMCI. Despite heterogeneity, MCI subtypes did have propensities for certain demographic and pathological measures. Individuals who came to autopsy with a diagnosis of aMCI during life were older at death, and were less likely to have executive dysfunction when compared to naMCI. Furthermore, amongst the plethora of pathologies, naMCI had a propensity for increased LBs and aMCI increased NFTs in select anatomic regions.
Table 3 Semi-quantitative analyses of neuritic plaques (NP) and neurofibrillary tangles (NFT), Lewy-type synucleinopathy (listed as average (mean)), and other pathologies (listed as frequencies) within $\mathrm{aMCl}(\mathrm{N}=34)$ and naMCl $(N=15)$

\begin{tabular}{|c|c|c|c|c|}
\hline & $\mathrm{aMCl}$ & $\mathrm{naMCl}$ & $95 \% \mathrm{Cl}$ & $p$-value \\
\hline \multicolumn{5}{|l|}{ Neuritic plaques } \\
\hline Frontal lobe & $1.5(1.3)$ & $1.8(1.3)$ & -1.0 to 0.6 & 0.55 \\
\hline Temporal lobe & $1.5(1.3)$ & $1.5(1.3)$ & -0.7 to 0.9 & 0.88 \\
\hline Parietal lobe & $1.6(1.3)$ & $1.7(1.7)$ & -1.0 to 0.7 & 0.76 \\
\hline Hippocampus region & $0.7(0.9)$ & $0.7(0.8)$ & -0.53 to 0.53 & 1.00 \\
\hline Entorhinal region & $1.3(1.3)$ & $1.3(1.0)$ & -0.8 to 0.8 & 0.97 \\
\hline $\begin{array}{l}\text { Total } \\
\text { (all areas; score out of 15) }\end{array}$ & $6.6(5.7)$ & $1.2(5.2)$ & -4.3 to 2.9 & 0.11 \\
\hline \multicolumn{5}{|l|}{ Neurofibrillary tangles } \\
\hline Braak NFT stage & IV $(\mid-\mathrm{VI})$ & III (II-IV) & -0.22 to 0.95 & 0.22 \\
\hline Frontal lobe & $0.4(0.6)$ & $0.2(0.3)$ & -0.06 to 0.60 & 0.11 \\
\hline Temporal lobe & $1(0.8)$ & $0.6(0.7)$ & -0.04 to 0.91 & 0.07 \\
\hline Parietal lobe & $0.4(0.6)$ & $0.1(0.2)$ & -0.02 to 0.60 & 0.07 \\
\hline Hippocampus region & $2.2(0.9)$ & $2.0(0.9)$ & -0.30 to 0.80 & 0.36 \\
\hline Entorhinal region & $2.6(0.8)$ & $2.5(0.8)$ & -0.38 to 0.60 & 0.66 \\
\hline $\begin{array}{l}\text { Total } \\
\text { (all areas; score out of 15) }\end{array}$ & $6.6(2.8)$ & $5.2(2.3)$ & -0.3 to 3.1 & 0.11 \\
\hline \multicolumn{5}{|l|}{ Lewy type synucleinopathy } \\
\hline Olfactory bulb & $1.1(1.6)$ & $1.5(1.8)$ & -1.5 to 0.7 & 0.44 \\
\hline Cingulate cortex & $0.6(1.1)$ & $1.2(1.6)$ & -1.4 to 0.1 & 0.11 \\
\hline Frontal lobe & $0.2(0.5)$ & $0.4(0.8)$ & -0.55 to 0.22 & 0.39 \\
\hline Temporal lobe & $0.2(0.5)$ & $0.7(1.0)$ & -0.9 to -0.3 & 0.04 \\
\hline Parietal lobe & $0.2(0.5)$ & $0.5(0.7)$ & -0.64 to 0.12 & 0.17 \\
\hline Amygdala & $0.9(1.5)$ & $1.5(1.8)$ & -1.5 to 0.5 & 0.29 \\
\hline Transentorhinal cortex & $0.6(1.1)$ & $1.1(1.5)$ & -1.3 to 0.3 & 0.23 \\
\hline $\begin{array}{l}\text { Total } \\
\text { (all areas; score out of 15) }\end{array}$ & $6.6(2.8)$ & $5.2(2.3)$ & -0.3 to3.1 & 0.11 \\
\hline \multicolumn{5}{|l|}{ Other pathologies } \\
\hline $\operatorname{Arg}$ & $13(37 \%)$ & $4(27 \%)$ & $\mathrm{n} / \mathrm{a}$ & 0.74 \\
\hline CWMR & $22(65 \%)$ & $10(67 \%)$ & $\mathrm{n} / \mathrm{a}$ & 0.89 \\
\hline CAA & $16(47 \%)$ & $10(67 \%)$ & $\mathrm{n} / \mathrm{a}$ & 0.21 \\
\hline Brain neoplasms & $2(6 \%)$ & 0 & $\mathrm{n} / \mathrm{a}$ & 1.0 \\
\hline Acute infarcts & $6(18 \%)$ & $2(13 \%)$ & $\mathrm{n} / \mathrm{a}$ & 1.0 \\
\hline
\end{tabular}

Although there have been an increased number of studies investigating the pathologic origins of MCI, these have mostly revolved around comparisons to cognitively normal elderly or those with AD or other dementias [5-13] with few studies directly comparing neuropathological differences between aMCI and naMCI. One of the best studies on this comparison was conducted through the Religious Orders Study/Rush Memory and Aging Project (ROS/MAP) which examined LBs, and AD pathologies in 75 individuals who died with aMCI and 59 with naMCI [10]. Although the ROS/MAP study did not describe 
Table 4 Infarcts (excluding acute) in aMCl and naMCl organized by location and size (microscopic (not grossly visible), lacunar $(<1 \mathrm{cc})$, small (1-27 cc), and large (>27 cc)). All data listed as N (\%). Groups are not mutually exclusive

\begin{tabular}{|c|c|c|c|c|c|}
\hline \multirow[b]{2}{*}{ Area affected } & \multicolumn{5}{|c|}{$\mathrm{aMCl}$} \\
\hline & Total cases & Microscopic & Lacunar & Small & Large \\
\hline Cortical & $10(29 \%)$ & $8(24 \%)$ & $2(6 \%)$ & $2(6 \%)$ & $2(6 \%)$ \\
\hline Centrum semiovale & $3(9 \%)$ & $2(6 \%)$ & $1(3 \%)$ & 1 (3 \%) & $1(3 \%)$ \\
\hline Deep nuclei & $12(35 \%)$ & $4(12 \%)$ & $9(26 \%)$ & $2(6 \%)$ & $1(3 \%)$ \\
\hline \multirow[t]{2}{*}{ Infratentorial } & $14(41 \%)$ & $12(35 \%)$ & $2(6 \%)$ & $2(6 \%)$ & $1(3 \%)$ \\
\hline & \multicolumn{5}{|c|}{ naMCl } \\
\hline Area affected & Total cases & Microscopic & Lacunar & Small & Large \\
\hline Cortical & $3(20 \%)$ & $1(7 \%)$ & 0 & $2(13 \%)$ & $1(7 \%)$ \\
\hline Centrum semiovale & $3(20 \%)$ & 0 & $2(13 \%)$ & 0 & $1(7 \%)$ \\
\hline Deep nuclei & $3(20 \%)$ & $2(13 \%)$ & $2(13 \%)$ & 0 & 0 \\
\hline Infratentorial & $3(20 \%)$ & $2(13 \%)$ & $1(7 \%)$ & 0 & 0 \\
\hline
\end{tabular}

anatomic distributions of LBs, AD pathologies, CAA, CWMR, or Arg, they did demonstrate that $59 \%$ of aMCI met pathological criteria for the diagnosis of AD compared to $49 \%$ of naMCI; these percentages are very similar to those presented in the current study. The ROS/ MAP study also had similar conclusion that many individuals with $\mathrm{MCI}$ exhibit mixed pathologies, thus emphasizing pathological heterogeneity.

In this cross-sectional study, although no global differences in $\mathrm{AD}$ pathology were found (i.e. meeting pathological criteria for $\mathrm{AD}$ ), certain anatomic regions (parietal and temporal lobes) had a propensity for increased NFTs in subjects with aMCI (p values $=0.07$ ). Furthermore, in both MCI groups, no cases were completely devoid of NFTs. In contrast, 26/50 (52 \%) of MCI cases were devoid of NPs. Given temporal lobe structures are affected early within the pathologic course of $\mathrm{AD}$ (as determined by Braak NFT stages) [25], this is in support of clinical studies having shown aMCI to have a greater conversion rate to probable AD than naMCI $[29,30]$. When examining MCI as one entity, several studies have described the presence of increased numbers of NFTs in subjects with MCI as compared with similarly-aged normal subjects, especially in medial temporal lobe structures [30,31]. Others have examined similarities in pathology between aMCI and $\mathrm{AD}$, suggesting that $\mathrm{aMCI}$ is identical to early $\mathrm{AD}$ based on NFT distribution [7, 9].

As for other neuropathological comparisons, a recent pathologic study demonstrated naMCI to have a greater conversion rate to dementia with LBs by time of autopsy [12]. With respect to PD, in a multicenter, pooled, clinically based study examining 347 PD with MCI, $49 \%$ had naMCI and $51 \%$ had aMCI [32]. Other data from the BBDP published on MCI within the setting of PD, too small to correlate pathologic and clinical findings, revealed varying LBs distribution [29]. In the current study, there was no large difference in the concurrency rate of clinicopathologically defined PD; however, Lewy-type alpha-

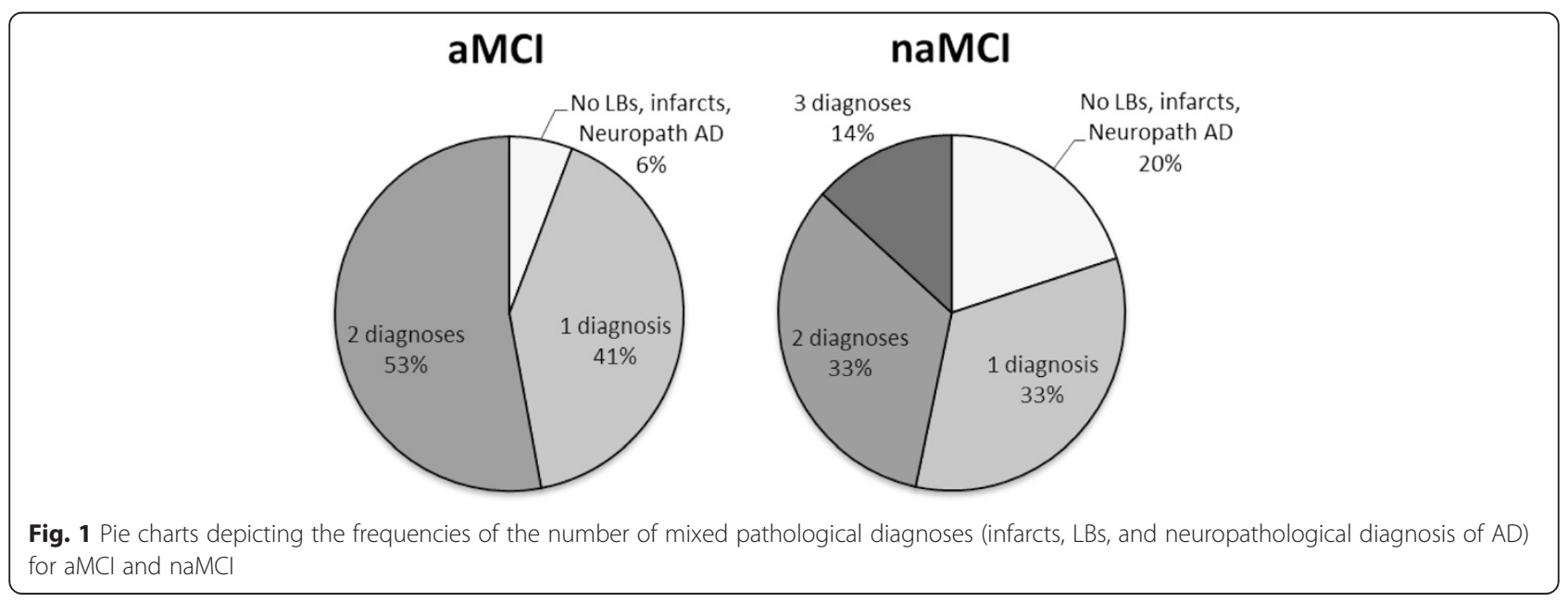


synucleinopathy had greater densities in the temporal lobe of naMCI. This distinct anatomic difference may be due to the temporal lobe (similar to data on NFTs) being affected somewhat early in the Lewy-type alpha-synuclein disease process [21].

Other pathologies, outside the realm of major clinicopathologically-defined conditions, such as CWMR, Arg, and CAA, may exist within MCI as well as within clinically normal elderly individuals. The most common pathology within the aMCI and naMCI groups were CWMR and CAA and both were equally common, although reports have demonstrated that CWMR and CAA have a greater association with AD [33-36]. Although no large associations were found with respect to amnestic type, there were associations with the executive dysfunction. Cases with executive dysfunction had larger frequencies of CWMR and CAA. A radiological study of white matter lesions in 129 patients demonstrated that the severity of white matter lesions was not associated with time to conversion to dementia for either MCI patients in general or amnestic MCI patients in particular [37]. Our frequencies of Arg in each subgroup are very similar to those that have been previously reported for the entire MCI entity [5].

One of the difficulties in conducting pathologic studies on MCI patients after death is that few autopsies have been done on individuals who died while still in the MCI stage; this is evident in the current study with less than $5 \%$ of the total number of BBDP participants having died in the MCI stage. Larger samples would be needed in order to detect or rule out smaller subtle differences between aMCI and naMCI. This study further emphasizes the dire need for in-vivo biomarkers that could demonstrate disease progression of LBs and NFTs, thus providing firmer conclusions. Individuals who die and undergo autopsy while in the MCI state may not represent the full spectrum of MCI due to survival bias, reversion back to normal cognition, and those who converted to dementia before death. One report revealed the mean time to developing dementia from MCI baseline was 3.1 years for those eventually diagnosed as clinically probable $\mathrm{AD}$, while this was 2.6 years for those eventually diagnosed with dementia with Lewy bodies [12]. Furthermore, cases included in this study were mainly elderly volunteers, typically over the age of 70 at enrollment. Due to the emphasis of the program being the study of normal aging, $\mathrm{PD}$, as well as dementia there is a recruitment bias towards enrolling subjects in these categories. Hence there may be an overrepresentation of Parkinsonian diagnoses in aMCI and naMCI- although no differences were seen between groups. Although this study had limitations, such as the small sample size and the greater proportion of aMCI cases, perhaps due to the known prevalence of MCI subtypes $[6,38]$, it is the most comprehensive study to date analyzing a full range of pathologies among amnestic and nonamnestic MCI subjects.

\section{Conclusions}

This small study suggests there may be no single underlying entity strongly associated with a specific domain deficit within $\mathrm{MCI}$, given the finding of multiple pathologies within aMCI and naMCI. Although there is a plethora of pathologies, naMCI had a propensity for an increased density of LBs and aMCI for increased NFTs in select anatomic regions. Furthermore, executive dysfunction in the setting of MCI was associated with the presence of CWMR and CAA.

\section{Competing interests}

B. Dugger has had a portion of her salary supported by Avid Radiopharmaceuticals, a division of Eli-Lily. K. Davis, M. Malek-Ahmadi, J.

Hentz, S. Sandhu, R. Caselli, H. Shill, T. Johnson, G. Serrano, L. Sue, J. Caviness, S. Jacobson, and J. Powell report no competing interests. C. Adler has had research funding from Avid Radiopharmaceuticals, a division of Eli-Lily. E. Driver-Dunckely has received clinical research funds from Ipsen and Chelsea. T. Beach receives consulting fees from GE Healthcare and Avid Radiopharmaceuticals. C. Belden is an investigator/rater for Piramal (Florbetaben), GE (Flutametamol), Avid (Florbetapir), Lilly, Functional Neuromodulation, Merck, Roche, Genetech, Navidea, DART, Neuronix, Takeda. M. Sabbagh is an investigator for Piramal (Florbetaben), GE (Flutametamol), Avid (Florbetapir), Lilly, Functional Neuromodulation, Merck, Roche, Genentech, Navidea, DART, Neuronix, Takeda. Dr Sabbagh serves as an advisor to Piramal, Lilly, Muses Labs, and Biogen.

\section{Authors' contributions}

$\mathrm{BD}$ oversaw the design of the study, aided in collection of data, statistical analyses, wrote the first draft of the manuscript and revised the manuscript. $\mathrm{JH}$, and MMA, aided in design of study, collection of data, statistical analyses, and drafting and critical revision of the manuscript for content. SS aided in the design of the study, drafting and critical revision of the manuscript for content. RC, TJ, CB, GS, KD, SJ, ED-D, JC, LS, HS and JP aided in collection of data for the study, and aided in critical revision of the manuscript. TB and CA, obtained funding, aided in data collections, and contributed to the critical revision of the manuscript for content. MS oversaw the design of the study, collection of data, drafting and critical revision of the manuscript. All authors read and approved the final manuscript.

\section{Acknowledgements}

The Brain and Body Donation Program is supported by the National Institute of Neurological Disorders and Stroke (U24 NS072026 National Brain and Tissue Resource for Parkinson's Disease and Related Disorders), the National Institute on Aging (P30 AG19610 Arizona Alzheimer's Disease Core Center), the Arizona Department of Health Services (contract 211002, Arizona Alzheimer's Research Center), the Arizona Biomedical Research Commission (contracts 4001, 0011, 05-901 and 1001 to the Arizona Parkinson's Disease Consortium) and the Michael J. Fox Foundation for Parkinson's Research.

\section{Author details}

${ }^{1}$ Civin Laboratory for Neuropathology, Banner Sun Health Research Institute, 10515 W. Santa Fe Dr., Sun City, AZ 85351, USA. ${ }^{2}$ The Cleo Roberts Center for Clinical Research, Banner Sun Health Research Institute, Sun City, AZ, USA. ${ }^{3}$ Mayo Clinic, Scottsdale, AZ, USA.

Received: 14 April 2015 Accepted: 10 August 2015 Published online: 20 August 2015

\section{References}

1. Petersen RC. Mild cognitive impairment as a diagnostic entity. J Intern Med. 2004;256(3):183-94. doi:10.1111/j.1365-2796.2004.01388.x.

2. Flicker C, Ferris SH, Reisberg B. Mild cognitive impairment in the elderly: predictors of dementia. Neurology. 1991;41(7):1006-9. 
3. Winblad B, Palmer K, Kivipelto M, Jelic V, Fratiglioni L, Wahlund LO, et al. Mild cognitive impairment-beyond controversies, towards a consensus: report of the International Working Group on Mild Cognitive Impairment. J Intern Med. 2004;256(3):240-6. doi:10.1111/j.1365-2796.2004.01380.x.

4. Albert MS, DeKosky ST, Dickson D, Dubois B, Feldman HH, Fox NC, et al. The diagnosis of mild cognitive impairment due to Alzheimer's disease: recommendations from the National Institute on Aging-Alzheimer's Association workgroups on diagnostic guidelines for Alzheimer's disease. Alzheimer Dement. 2011;7(3):270-9. doi:10.1016/j.jalz.2011.03.008.

5. Saito $Y$, Murayama S. Neuropathology of mild cognitive impairment. Neuropathology. 2007;27(6):578-84. doi:10.1111/j.1440-1789.2007.00806.x.

6. Markesbery WR. Neuropathologic alterations in mild cognitive impairment: a review. J Alzheimer Dis. 2010;19(1):221-8. doi:10.3233/JAD-2010-1220.

7. Markesbery WR, Schmitt FA, Kryscio RJ, Davis DG, Smith CD, Wekstein DR. Neuropathologic substrate of mild cognitive impairment. Arch Neurol. 2006;63(1):38-46. doi:10.1001/archneur.63.1.38.

8. Bennett DA, Schneider JA, Bienias JL, Evans DA, Wilson RS. Mild cognitive impairment is related to Alzheimer disease pathology and cerebral infarctions. Neurology. 2005;64(5):834-41. doi:10.1212/ 01.WNL.0000152982.47274.9E.

9. Petersen RC, Parisi JE, Dickson DW, Johnson KA, Knopman DS, Boeve BF et al. Neuropathologic features of amnestic mild cognitive impairment. Arch Neurol. 2006;63(5):665-72. doi:10.1001/archneur.63.5.665.

10. Schneider JA, Arvanitakis Z, Leurgans SE, Bennett DA. The neuropathology of probable Alzheimer disease and mild cognitive impairment. Ann Neurol. 2009;66(2):200-8. doi:10.1002/ana.21706.

11. Jicha GA, Parisi JE, Dickson DW, Johnson K, Cha R, Ivnik RJ, et al. Neuropathologic outcome of mild cognitive impairment following progression to clinical dementia. Arch Neurol. 2006;63(5):674-81. doi:10.1001/archneur.63.5.674

12. Ferman TJ, Smith GE, Kantarci K, Boeve BF, Pankratz VS, Dickson DW, et al. Nonamnestic mild cognitive impairment progresses to dementia with Lewy bodies. Neurology. 2013;81(23):2032-8. doi:10.1212/ 01.wnl.0000436942.55281.47.

13. Galvin JE, Powlishta KK, Wilkins K, McKeel Jr DW, Xiong C, Grant E, et al. Predictors of preclinical Alzheimer disease and dementia: a clinicopathologic study. Arch Neurol. 2005;62(5):758-65. doi:10.1001/ archneur.62.5.758.

14. Beach TG, Sue LI, Walker DG, Roher AE, Lue L, Vedders $L$, et al. The Sun Health Research Institute Brain Donation Program: description and experience, 1987-2007. Cell Tissue Bank. 2008;9(3):229-45. doi:10.1007/ s10561-008-9067-2

15. Beach TG, Adler CH, Sue LI, Serrano G, Shill HA, Walker DG, et al. Arizona study of aging and neurodegenerative disorders and brain and body donation program. Neuropathology. 2015;35:354-89. doi:10.1111/ neup.12189.

16. Ellison D. Neuropathology: a reference text of CNS pathology. 2nd ed. Edinburgh, New York: Mosby; 2004.

17. Dugger BN, Hentz JG, Adler CH, Sabbagh MN, Shill HA, Jacobson S, et al. Clinicopathological outcomes of prospectively followed normal elderly brain bank volunteers. J Neuropathol Exp Neurol. 2014;73(3):244-52. doi:10.1097/NEN.0000000000000046.

18. Hixson JE, Vernier DT. Restriction isotyping of human apolipoprotein E by gene amplification and cleavage with Hhal. J Lipid Res. 1990;31(3):545-8.

19. Beach TG, Sue LI, Walker DG, Sabbagh MN, Serrano G, Dugger B, et al. Striatal amyloid plaque density predicts Braak neurofibrillary stage and clinicopathological Alzheimer's disease: implications for amyloid imaging. J Alzheimers Dis. 2011;28:869-76. doi:10.3233/JAD-2011-111340.

20. Braak H, Braak E. Demonstration of amyloid deposits and neurofibrillary changes in whole brain sections. Brain Pathol. 1991;1(3):213-6.

21. Beach TG, Adler CH, Lue L, Sue LI, Bachalakuri J, Henry-Watson J, et al. Unified staging system for Lewy body disorders: correlation with nigrostriatal degeneration, cognitive impairment and motor dysfunction. Acta Neuropathologica. 2009;117(6):613-34. doi:10.1007/s00401-009-0538-8.

22. Beach TG, White $\mathrm{CL}$, Hamilton RL, Duda JE, Iwatsubo T, Dickson DW, et al. Evaluation of alpha-synuclein immunohistochemical methods used by invited experts. Acta Neuropathologica. 2008;116(3):277-88. doi:10.1007/ s00401-008-0409-8.

23. Mirra SS, Heyman A, McKeel D, Sumi SM, Crain BJ, Brownlee LM, et al. The Consortium to Establish a Registry for Alzheimer's Disease (CERAD). Part II.
Standardization of the neuropathologic assessment of Alzheimer's disease. Neurology. 1991;41(4):479-86.

24. Consensus recommendations for the postmortem diagnosis of Alzheimer's disease. The National Institute on Aging, and Reagan Institute Working Group on Diagnostic Criteria for the Neuropathological Assessment of Alzheimer's Disease. Neurobiol Aging. 1997;18(4 Suppl):S1-2.

25. Braak H, Braak E. Neuropathological stageing of Alzheimer-related changes. Acta Neuropathologica. 1991;82(4):239-59.

26. Dugger BN, Clark CM, Serrano G, Mariner M, Bedell BJ, Coleman RE, et al. Neuropathologic heterogeneity does not impair florbetapir-positron emission tomography postmortem correlates. J Neuropathol Exp Neurol. 2014;73(1):72-80. doi:10.1097/NEN.0000000000000028.

27. Sabbagh MN, Sandhu SS, Farlow MR, Vedders L, Shill HA, Caviness JN, et al. Correlation of clinical features with argyrophilic grains at autopsy. Alzheimer Dis Assoc Disord. 2009;23(3):229-33. doi:10.1097/WAD.0b013e318199d833.

28. Schneider JA, Arvanitakis Z, Bang W, Bennett DA. Mixed brain pathologies account for most dementia cases in community-dwelling older persons. Neurology. 2007;69(24):2197-204. doi:10.1212/01.wnl.0000271090.28148.24.

29. Adler CH, Caviness JN, Sabbagh MN, Shill HA, Connor DJ, Sue L, et al. Heterogeneous neuropathological findings in Parkinson's disease with mild cognitive impairment. Acta Neuropathologica. 2010;120(6):827-8. doi:10.1007/s00401-010-0744-4.

30. Guillozet AL, Weintraub S, Mash DC, Mesulam MM. Neurofibrillary tangles, amyloid, and memory in aging and mild cognitive impairment. Arch Neurol. 2003;60(5):729-36. doi:10.1001/archneur.60.5.729.

31. Sabbagh MN, Shah F, Reid RT, Sue L, Connor DJ, Peterson LK, et al. Pathologic and nicotinic receptor binding differences between mild cognitive impairment, Alzheimer disease, and normal aging. Arch Neurol. 2006;63(12):1771-6. doi:10.1001/archneur.63.12.1771.

32. Aarsland D, Bronnick K, Williams-Gray C, Weintraub D, Marder K, Kulisevsky J, et al. Mild cognitive impairment in Parkinson disease: a multicenter pooled analysis. Neurology. 2010;75(12):1062-9. doi:10.1212/ WNL.0b013e3181f39d0e.

33. White $L$, Launer $L$. Relevance of cardiovascular risk factors and ischemic cerebrovascular disease to the pathogenesis of Alzheimer disease: a review of accrued findings from the Honolulu-Asia Aging Study. Alzheimer Dis Assoc Disord. 2006;20(3 Suppl 2):S79-83.

34. Hirono N, Kitagaki H, Kazui H, Hashimoto M, Mori E. Impact of white matter changes on clinical manifestation of Alzheimer's disease: A quantitative study. Stroke. 2000;31(9):2182-8.

35. Roher AE, Kuo YM, Esh C, Knebel C, Weiss N, Kalback W, et al. Cortical and leptomeningeal cerebrovascular amyloid and white matter pathology in Alzheimer's disease. Mol Med. 2003;9(3-4):112-22.

36. Honig LS, Kukull W, Mayeux R. Atherosclerosis and AD: analysis of data from the US National Alzheimer's Coordinating Center. Neurology. 2005;64(3):494-500. doi:10.1212/01.WNL.0000150886.50187.30.

37. Devine ME, Fonseca JA, Walker Z. Do cerebral white matter lesions influence the rate of progression from mild cognitive impairment to dementia? Int Psychogeriatr. 2013;25(1):120-7. doi:10.1017/ S1041610212000932

38. Petersen RC, Negash S. Mild cognitive impairment: an overview. CNS Spectr. 2008;13(1):45-53.

\section{Submit your next manuscript to BioMed Central and take full advantage of:}

- Convenient online submission

- Thorough peer review

- No space constraints or color figure charges

- Immediate publication on acceptance

- Inclusion in PubMed, CAS, Scopus and Google Scholar

- Research which is freely available for redistribution

Submit your manuscript at www.biomedcentral.com/submit 